\title{
CARACTERIZAÇÃO DE FRUTOS E ÁRVORES DE CAGAITA (Eugenia dysenterica DC.) NO SUDESTE DO ESTADO DE GOIÁS, BRASIL ${ }^{1}$
}

\author{
ROSSANA SERRATO MENDONÇA SILVA², LÁZARO JOSÉ CHAVES ${ }^{3}$ E RONALDO VELOSO NAVES 4
}

\begin{abstract}
RESUMO - Dentre as espécies frutíferas do bioma cerrado destaca-se a cagaiteira (Eugenia dysenterica DC., Myrtaceae), cujos frutos são consumidos in natura ou processados. Os frutos, folhas e casca apresentam propriedades medicinais, a madeira é utilizada em pequenas construções e carvão. Objetivou-se caracterizar frutos e árvores da espécie por amostragem de populações de plantas existentes no Sudeste de Goiás. Coletaram-se 1344 frutos de 112 plantas de 10 sub-populações para caracterização física dos mesmos e dados de 95 árvores, visando a sua descrição morfológica. Constatou-se variação significativa ao nível de $1 \%$ de probabilidade entre as 112 plantas para todos os caracteres de fruto, assim como entre plantas dentro de subpopulação e entre as médias de subpopulações. A média para a variável peso de fruto e número de sementes por fruto foi de $12,67 \mathrm{~g}$ e 1,70 , respectivamente. Os valores máximos e mínimos encontrados para a variável altura de planta foram, respectivamente, $11,0 \mathrm{~m}$ e 4,10 $\mathrm{m}$ e para diâmetro de copa foram de $10,30 \mathrm{~m}$ e $1,80 \mathrm{~m}$.
\end{abstract}

Termos para indexação: Eugenia dysenterica, cagaita, cerrado, variação morfométrica

\section{CARACTERIZATION OF CAGAITA (Eugenia dysenterica DC.) FRUITS AND TREE IN SOUTHEASTERN REGION OF GOIAS STATE, BRAZIL}

\begin{abstract}
Cagaita, Eugenia dysenterica DC., Myrtaceae, stands out among the fruit tree species in the savanna ecosystem. Fruits of this species are consumed fresh or processed, the leaves, fruits and bark have medicinal proprieties and the wood is utilized for small construction and charcoal. Our objective was the characterization the trees and fruits of the species by sampling the populations found in Southeastern Goias State. Fruit characteristics were evaluated for 1344 fruits from 112 plants from 10 sub-populations, and data from 95 plants from ten sub-populations were collected for morphological description. Fruit characteristics showed significant variation at the $1 \%$ probability level among the 112 plants, as well as within the sub-populations and among sub-population means. The average fruit weight and seed number per fruit were 12.67 and 1.7 respectively. The highest and lowest values for plant height were $11.0 \mathrm{~m}$ and $4.10 \mathrm{~m}$, where as for crown diameter were $10.3 \mathrm{~m}$ and $1.30 \mathrm{~m}$ respectively.
\end{abstract}

Index terms: Eugenia dysenterica, cagaita, savanna, morphometric variation

\section{INTRODUÇÃO}

Eugenia dysenterica DC. (Myrtaceae), popularmente conhecida como "cagaita" ou "cagaiteira", é uma árvore frutífera nativa dos cerrados de até $10 \mathrm{~m}$ de altura, de tronco e ramos tortuosos, casca grossa, fissurada. Os frutos têm formato globoso, bagáceo, cor amarelo-clara, levemente ácido, epicarpo membranoso, com peso entre 14 a $20 \mathrm{~g}$, comprimento de 3 a $4 \mathrm{~cm}$ e diâmetro de 3 a 5 cm, (Rizzini, 1971; Ribeiro et al., 1986; Naves et al., 1995) (Figura 1). A cagaiteira apresenta tanto autofecundação quanto fecundação cruzada, sendo a polinização realizada principalmente pelas mamangavas das espécies Bombus atratus e B. morio, no período da manhã (Proença \& Gibbs, 1994). Ocorre em áreas de cerrado e cerradão, estando adaptada a solos pobres; portanto, supõe-se que é pouco exigente em fertilidade (Naves, 1999). A cagaita foi estudada, destacando-se germinação e armazenamento de sementes (Machado et al., 1986; Farias Neto et al., 1991; Naves et al., 1992), fenologia e estratégia de florescimento (Proença \& Gibbs, 1994; Sano et al., 1995), caracterização e armazenamento de frutos (Rizzini, 1971; Calbo et al.,1990; Silva et al.,1992).

Os frutos de cagaita são consumidos in natura ou processados (licor, sorvete, suco, geléia). Esta espécie faz parte da flora apícola do cerrado e suas folhas e cascas são utilizadas na medicina popular como antidiarréico, para diabete e icterícia. Tem potencial para utilização como planta ornamental, como fornecedora de cortiça e sua madeira pode ser empregada para obras da construção civil, lenha e carvão (Brandão \& Ferreira, 1991; Ribeiro et al., 1992).

Partindo da hipótese de que existe variabilidade disponível para ser explorada em programas de seleção para caracteres de frutos e plantas, objetivou-se, neste trabalho, obter informações sobre a variabilidade existente em cagaita, no Sudeste de Goiás, avaliando as características físicas dos frutos e descrevendo as árvores quanto a alguns aspectos morfológicos

\footnotetext{
1 Trabalho $n^{\circ}$ 125/2000. Recebido: 05/07/2000. Aceito para publicação: 27/06/2001. Parte de Dissertação de Mestrado apresentada pela primeira autora junto à Universidade Federal de Goiás

2 Engenheira Agrônoma MS. - Bolsista - CNPq/EMBRAPA/SNT - CP. 714, CEP 74000.970, Goiânia - GO, Email: spsbgyn@zaz.com.br

3 Engenheiro Agrônomo Doutor, Professor Titular da Escola de Agronomia da Universidade Federal de Goiás - CP.131, CEP 74001.970, GoiâniaGO,Email: lchaves@agro.ufg.br

4 Engenheiro Agrônomo Doutor, Professor Adjunto da Escola de Agronomia da Universidade Federal de Goiás - CP.131, CEP 74001.970, GoiâniaGO, Email: ronaldo@agro.ufg.br
} 


\section{MATERIAL E MÉTODOS}

Selecionaram-se 10 áreas no Sudeste do Estado de Goiás, compreendidas entre as latitudes $16^{\circ} 28^{\prime} 48^{\prime \prime} \mathrm{S}$ a $18^{\circ} 17^{\prime} 15^{\prime \prime} \mathrm{S}$ e longitudes $47^{\circ} 31^{\prime} 07^{\prime \prime} \mathrm{W}$ a $49^{\circ} 14^{\prime} 42^{\prime \prime} \mathrm{W}$ e entre as altitudes $740 \mathrm{~m}$ a $930 \mathrm{~m}$, considerando-se como propícia para coleta de dados aquelas que possuíssem no mínimo 20 plantas adultas de cagaiteira em um raio de 1,0 km. Denominou-se "subpopulação" o conjunto de plantas amostradas, levando-se em consideração uma distância mínima de cerca de $20 \mathrm{~km}$ entre áreas.

Coletaram-se, em outubro de 1996, frutos para caracterização física, com o seguinte procedimento: os galhos de cada árvore foram balançados, aparando-se os frutos com auxílio de uma rede colocada sob sua copa; esta operação foi repetida nos quatro quadrantes de cada árvore, formando-se uma amostra composta. Uma subamostra contendo 12 frutos intactos e individualizados por árvore foi retirada aleatoriamente desta amostra.

No Laboratório de Fitotecnia, da Escola de Agronomia da Universidade Federal de Goiás, em Goiânia-GO, realizaram-se as análises físicas individualizadas por fruto: peso dos frutos (PF) e das sementes (PS); diâmetros longitudinal (DLF) e transversal do fruto (DTF); cor do fruto (CF), sendo avalianda a coloração externa do fruto por meio de uma escala de cores feita com seis frutos, atribuindo-se notas de 1 (completamente amarelo) a 6 (completamente verde) e número de semente por fruto (NS); o peso total de sementes (PTS); peso médio de sementes por fruto (PMS) obtido a partir da pesagem individual das sementes e o peso de polpa e casca (PPC), obtido através da diferença entre o peso do fruto e o peso da(s) semente(s).

Coletou-se das árvores dados relativos às variáveis: altura da planta (AP), diâmetro de projeção da copa nos sentidos nortesul e leste-oeste, obtendo-se diâmetro médio de copa (DMC) e área basal $(\mathrm{ABa})$, calculada a partir da circunferência do colo ao nível do solo.

Para as variáveis dos frutos, além da estimativa da média, coeficiente de variação e intervalo de variação, procedeu-se à análise de variância, utilizando-se como repetições dos dados de frutos dentro de plantas, a partir de um modelo hierárquico que considera os efeitos entre subpopulações, plantas dentro de subpopulações e frutos dentro de plantas. Calcularam-se também os coeficientes de correlação fenotípica entre as variáveis de fruto a partir das médias das plantas. Para as variáveis das árvores, estimaram-se a média, coeficiente de variação e intervalo de variação, considerando-se os dados gerais e por subpopulação.

\section{RESULTADOS E DISCUSSÃO}

Vinte e três por cento dos frutos mostraram-se próximos ao intervalo de 14 a 20 g encontrado por Silva et al. (1992) na região de Planaltina-DF, e 53\% entre o intervalo de 11 a 33,8 g, citado por Naves et al. (1995), de uma população de plantas de Senador Canedo-GO. Os intervalos de variação para diâmetro longitudinal de fruto (DLF) e diâmetro transversal de fruto (DTF) foram maiores do que os encontrados por Ribeiro et al. (1985), na região de Planaltina-DF, e Naves et al. (1995). Com relação ao formato dos frutos, este apresentou, em geral, DLF menor do que DTF, apresentando na média por subpopulação razão DLF/ DTF de 0,84 , com variação de 0,80 a 0,86 , tendo como característica aspecto de fruto achatado ou globoso (Tabela 1).

Dentre todos os caracteres físicos de fruto, o peso de polpa e casca (PPC) apresentou-se como o de maior importância para exploração econômica, principalmente processamento de frutos, pois é esta a parte aproveitável do fruto. Nas populações avaliadas, o PPC variou de $80 \%$ a $87 \%$ do peso do fruto e com média de $83,2 \%$, mostrando a possibilidade de seleção de frutos com maior PPC e, conseqüentemente, alto rendimento de polpa para indústria.

O número de sementes (NS) por fruto foi bastante variável, como citam Ribeiro et al. (1985) e Silva et al. (1992). Cerca de $97 \%$ dos 1344 frutos observados apresentaram de 1 a 3 sementes/ fruto, similar aos valores relatados pelos autores referidos. Rizzini (1970) relatou a presença de frutos contendo 5 sementes, observado, neste trabalho, em 2 frutos. Constatou-se, ainda, a presença de 1 fruto com 6 sementes, fato não relatado na literatura pesquisada. $\mathrm{O}$ peso da semente representa, em média, 16\% do peso total do fruto.

Observou-se que, para todos os caracteres de frutos, a variação entre as plantas foi significativa, bem como a variação entre e dentro de subpopulações. Telles (2000) analisou progênies das mesmas subpopulações utilizando marcadores enzimáticos. Os resultados encontrados mostraram que variação entre subpopulações em relação à população total foi de $16,4 \%$. Isto mostra a existência de diferenças genéticas entre as subpopulações. A variação fenotípica existente deve ser bastante influenciada por componentes ambientais não controlados, tais como a condição de antropização, o solo, o clima, a idade da planta, além das diferenças genéticas.

$\mathrm{O}$ peso de fruto apresentou correlação positiva e significativa ao nível de $1 \%$ de probabilidade, com as seguintes variáveis: $\operatorname{DLF}(0,8964), \operatorname{DTF}(0,9809), \operatorname{PPC}(0,9943), \operatorname{PTS}(0,6292)$ e PMS (0,6225). A variável NS apresentou correlação negativa e significativa em nível de $1 \%$ de probabilidade com PMS (-0,5472). A correlação entre PTS e NS (0,2176) apresentou-se positiva e significativa a nível de $5 \%$ de probabilidade. O número de sementes independe do tamanho do fruto, pois as variáveis que indicam tamanho, peso de fruto e número de sementes por fruto, apresentaram correlação não significativa. A cor dos frutos mostrou variação independente de todas as demais variáveis, não sendo influenciada pelo tamanho dos frutos. Isto mostra que alguns frutos, mesmo já tendo atingido a maturação completa, ainda não tinham iniciado o processo de amadurecimento.

O intervalo de variação para a altura de planta (Tabela 2) foi superior ao citado por Rizzini (1971). As plantas da subpopulação 9 apresentaram altura média bem superior às demais, provavelmente devido a esta subpopulação estar localizada em área urbana, com forte alteração antrópica, onde as árvores se encontravam isoladas, e sem competição por água, luz e nutrientes. As subpopulações 1 e 8 encontravam-se em áreas de cerrado stricto sensu, sem alteração antrópica marcante, e a altura média das plantas avaliadas nestas áreas não diferiram da citada em literatura para este tipo fisionômico de cerrado (Naves et al., 1995), apresentando valores pouco abaixo das demais áreas. As demais subpopulações encontravam-se em áreas com diferentes graus de antropização, geralmente em pastagens "sujas" com presença de capim-braquiária (Brachiaria 
TABELA 1 - Médias e coeficientes de variação por subpopulação, valores máximo e mínimo e média geral das variáveis peso de fruto (PF), diâmetro longitudinal de fruto (DLF), diâmetro transversal de fruto (DTF), peso de polpa + casca (PPC) número de sementes/fruto (NS), peso total de sementes/fruto (PTS), peso médio de sementes/fruto (PMS) e cor de fruto (CF) de 10 subpopulações de cagaita do Sudeste de Goiás, 1997.

\begin{tabular}{|c|c|c|c|c|c|c|c|c|c|c|c|c|c|c|c|c|c|}
\hline \multirow{2}{*}{ Sub-pop. } & \multirow{2}{*}{$\begin{array}{c}\text { Número de } \\
\text { plantas }\end{array}$} & \multicolumn{2}{|c|}{$\mathrm{PF}(\mathrm{g})$} & \multicolumn{2}{|c|}{$\mathrm{DLF}(\mathrm{mm})$} & \multicolumn{2}{|c|}{$\mathrm{DTF}(\mathrm{mm})$} & \multicolumn{2}{|c|}{ PPC (g) } & \multicolumn{2}{|c|}{ NS } & \multicolumn{2}{|c|}{ PTS (g) } & \multicolumn{2}{|c|}{ PMS (g) } & \multicolumn{2}{|c|}{$\mathrm{CF}$} \\
\hline & & Méd. & $\mathrm{CV}(\%)$ & Méd. & CV (\%) & Méd. & CV (\%) & Méd. & $\mathrm{CV}(\%)$ & Méd. & CV (\%) & Méd. & $\mathrm{CV}(\%)$ & Méd. & CV (\%) & Méd. & CV (\%) \\
\hline 1 & 12 & 11,04 & 37,3 & 23,31 & 14,4 & 27,21 & 13,4 & 9,22 & 39,0 & 1,72 & 51,0 & 1,83 & 44,3 & 1,15 & 33,7 & 1,67 & 81,4 \\
\hline 2 & 12 & 13,70 & 46,0 & 24,33 & 18,8 & 29,65 & 16,5 & 11,59 & 47,6 & 1,44 & 48,9 & 2,11 & 54,0 & 1,52 & 39,1 & 2,69 & 68,3 \\
\hline 3 & 12 & 17,09 & 37,3 & 25,76 & 11,1 & 32,26 & 13,9 & 14,68 & 39,2 & 1,73 & 45,5 & 2,41 & 44,3 & 1,47 & 28,6 & 2,22 & 60,8 \\
\hline 4 & 12 & 15,12 & 50,0 & 26,30 & 12,1 & 30,89 & 13,9 & 12,74 & 40,7 & 1,81 & 44,1 & 2,38 & 45,9 & 1,36 & 27,4 & 3,56 & 49,5 \\
\hline 5 & 12 & 11,67 & 39,0 & 23,40 & 11,8 & 27,97 & 14,7 & 10,11 & 41,9 & 1,60 & 49,8 & 1,56 & 42,7 & 1,08 & 42,4 & 2,50 & 68,9 \\
\hline 6 & 12 & 11,99 & 36,5 & 23,89 & 13,4 & 28,66 & 12,6 & 9,55 & 37,3 & 2,08 & 46,3 & 2,04 & 46,0 & 1,06 & 38,7 & 2,99 & 67,1 \\
\hline 7 & 8 & 10,18 & 29,3 & 23,36 & 12,1 & 27,11 & 10,7 & 8,28 & 30,2 & 1,76 & 47,9 & 1,90 & 39,7 & 1,18 & 31,4 & 4,03 & 34,4 \\
\hline 8 & 12 & 8,80 & 33,1 & 21,77 & 11,8 & 26,08 & 11,3 & 7,13 & 34,7 & 1,69 & 43,2 & 1,67 & 42,8 & 1,04 & 31,7 & 4,65 & 20,3 \\
\hline 9 & 8 & 12,65 & 37,5 & 24,34 & 14,7 & 28,69 & 14,1 & 10,19 & 43,9 & 1,33 & 43,1 & 2,46 & 41,9 & 1,91 & 29,3 & 3,10 & 56,0 \\
\hline 10 & 12 & 13,60 & 46,5 & 24,02 & 16,8 & 29,66 & 16,2 & 11,29 & 51,1 & 1,72 & 48,6 & 2,31 & 39,8 & 1,50 & 39,2 & 2,47 & 82,7 \\
\hline \multicolumn{2}{|c|}{ Valor máx. } & \multicolumn{2}{|c|}{41,95} & \multicolumn{2}{|c|}{38,06} & \multicolumn{2}{|c|}{46,45} & \multicolumn{2}{|c|}{36,03} & \multicolumn{2}{|c|}{6} & \multicolumn{2}{|c|}{7,52} & \multicolumn{2}{|c|}{3,58} & \multicolumn{2}{|c|}{6} \\
\hline \multicolumn{2}{|c|}{ Valor mín. } & \multicolumn{2}{|c|}{2,95} & \multicolumn{2}{|c|}{12,00} & \multicolumn{2}{|c|}{17,60} & \multicolumn{2}{|c|}{2,03} & \multicolumn{2}{|c|}{1} & \multicolumn{2}{|c|}{0,07} & \multicolumn{2}{|c|}{0,07} & \multicolumn{2}{|c|}{1} \\
\hline \multicolumn{2}{|c|}{ Média geral } & \multicolumn{2}{|c|}{12,67} & \multicolumn{2}{|c|}{24,05} & \multicolumn{2}{|c|}{28,88} & \multicolumn{2}{|c|}{10,61} & & 70 & & & &, 31 & & 95 \\
\hline
\end{tabular}

TABELA 2 - Média e coeficiente de variação (CV) para a variável altura de planta (AP), diâmetro médio de copa (DMC) e área basal (ABa) de 95 árvores de cagaita de 10 subpopulações do Sudeste de Goiás, 1997.

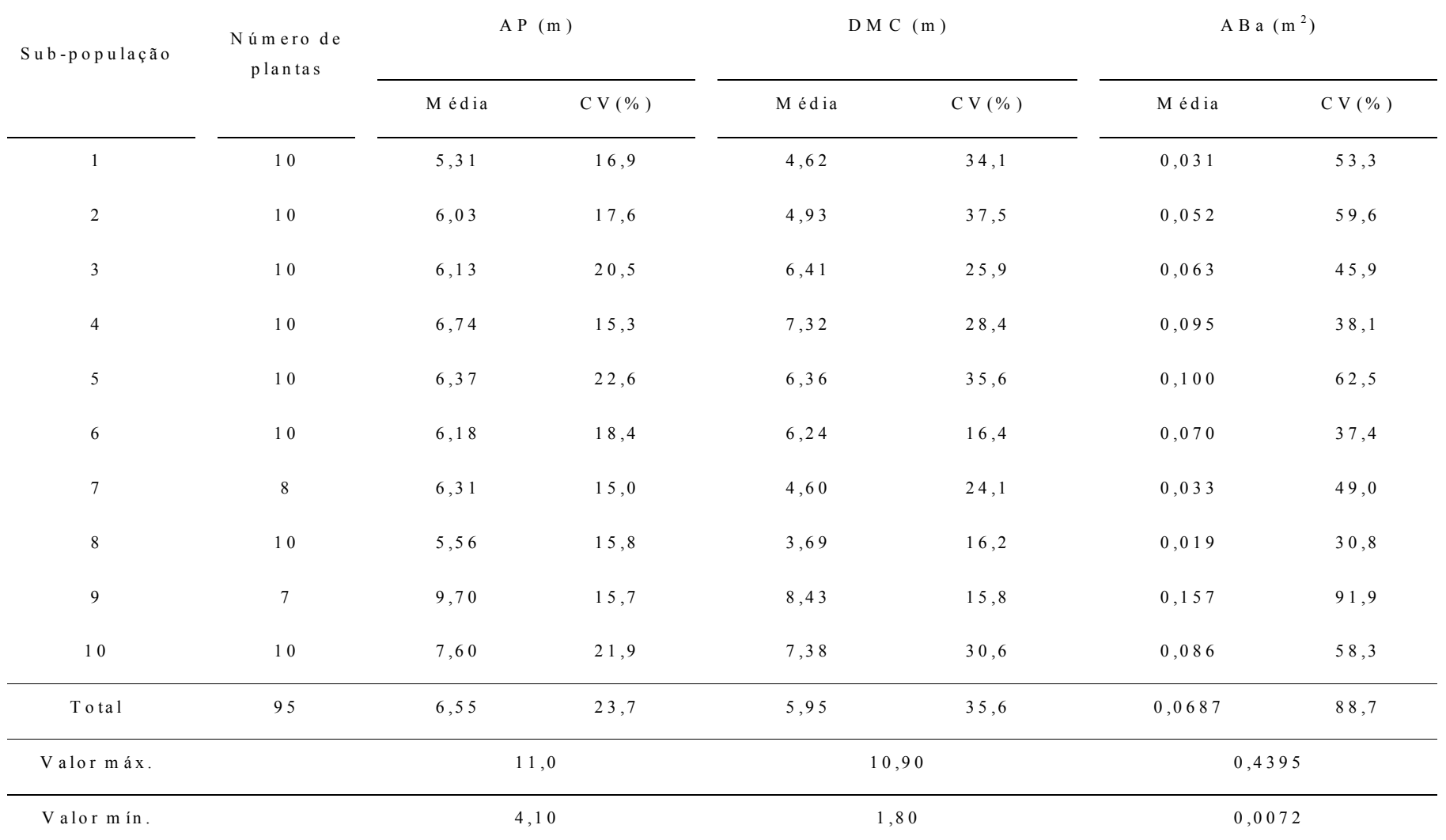




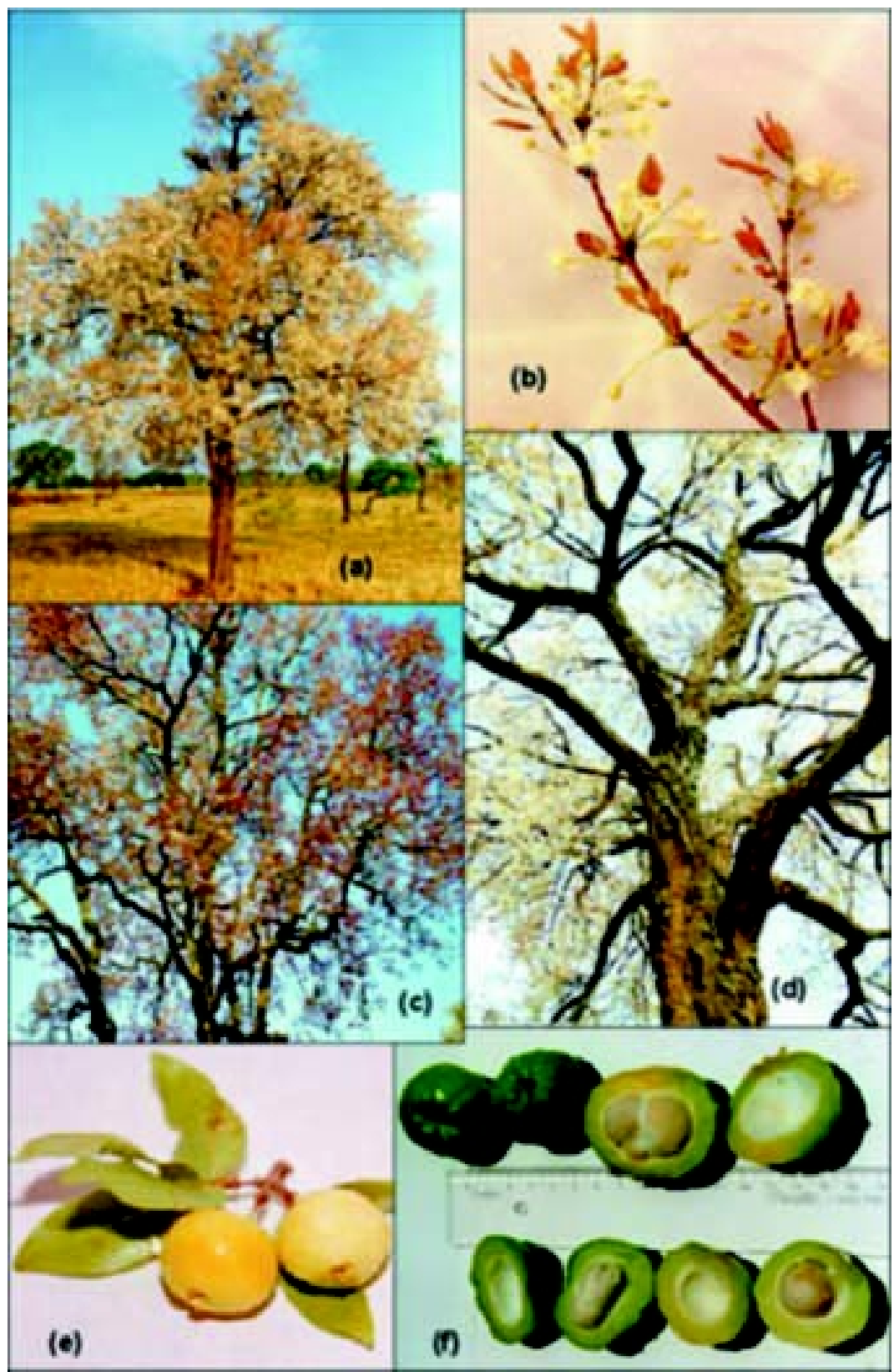

FIGURA 1 - Árvore de cagaita em florescimento (a). Detalhe da inflorescência (b). Cagaiteira com folhas novas (c). Detalhe do tronco (d). Fruto maduro e folha (e). Fruto verde e semente (f).

decumbens), onde não foram executadas roçagens freqüentes.

Vinte porcento das árvores apresentaram diâmetro médio de copa (DMC) acima de $8,00 \mathrm{~m}$, valor maior que os relatados em pesquisas anteriores. As subpopulações 1 e 8 apresentaram as menores médias de DMC, mostrando mais uma vez o efeito da competição em áreas pouco antropizadas. A média de área basal (ABa) foi de $0,0687 \mathrm{~m}^{2}$, o que corresponde a $0,86 \mathrm{~m}$ de circunferência de tronco. Os valores encontrados para esta variável foram semelhantes aos encontrados por Ribeiro et al. (1985) para áreas de cerrado em Planaltina-DF. Nas áreas com pequena ação antrópica (subpopulações 1 e 8 e parte da subpopulação 7), as cagaiteiras apresentaram menor área basal; o contrário pode ser observado em áreas mais antropizadas (subpopulações 4; 5; 9 e 10), onde os exemplares se encontravam mais isolados. De acordo com Naves (1999), as plantas de cagaita mostram certa exigência por luminosidade, visto pela maior área basal quando encontradas em áreas mais abertas.

\section{CONCLUSÕES}

1. Existe variação fenotípica entre subpopulações e entre plantas dentro de subpopulação para os caracteres morfométricos de frutos.

2. A cagaita mostra grande variação quanto às características morfológicas das árvores.

3. Os frutos de maior peso ou tamanho serão preferidos para industrialização por apresentarem maior peso de polpa e casca, conseqüentemente, maior rendimento no processamento. 


\section{REFERÊNCIAS BIBLIOGRÁFICAS}

BRANDÃO, M.; FERREIRA, P.B.D. Flora apícola do cerrado. Informe Agropecuário, Belo Horizonte, v.15, n.168, p.7-14, 1991.

CALBO, M.E.R.; LIMA, J.N.C.; CALBO, A.G. Fisiologia póscolheita de frutos de cagaita. Revista Brasileira de Fisiologia Vegetal, Brasília, v.2, n.1, p.15-18, 1990.

FARIAS NETO, A.L. de; FONSECA, C.E.L. da; GOMIDE, C.C.C. et al. Armazenamento de sementes de cagaita (Eugenia dysenterica DC.). Revista Brasileira de Fruticultura, Cruz das Almas, v.13, n.2, p.55-62, 1991.

MACHADO, J.W.B.; PARENTE, T.V.; LIMA, R.M. Informações sobre germinação e características físicas das sementes de fruteiras nativas do Distrito Federal. Revista Brasileira de Fruticultura, Cruz das Almas, v.8, n.2, p.59-62, 1986.

NAVES, R.V.; BORGES, J.D.; ROCHA, M.R.; CHAVES, L.J. et al. Emergência de plântulas de cagaita (Eugenia dysenterica DC) em viveiro. Revista Brasileira de Fruticultura, Cruz das Almas, v.14, n.2, p.37-40, 1992.

NAVES, R.V.; ALMEIDA NETO, J.X.; ROCHA, M.R.; et al. Determinação de características físicas em frutos e teor de nutrientes em folhas e no solo, de três espécies frutíferas de Ocorrência Natural nos Cerrados de Goiás. Anais da Escola de Agronomia e Veterinária, Goiânia, v.25, p. 99-106, 1995.

NAVES, R.V. Espécies frutíferas nativas do cerrado de Goiás: caracterização e influências do clima e dos solos. 1999. 206 f. Tese (Doutorado), Escola de Agronomia, Universidade Federal de Goiás, Goiânia, 1999.
PROENÇA, C.E.B.; GIBBS, P.E. Reproductive biology of eight sympatric Myrtaceae from Central Brazil. New Phytologist, Oxford, v.126, p.343-354, 1994.

RIBEIRO, J.F.; SILVA, J.C.S.; BATMANIAN, G.J. Fitossociologia de tipos de cerrado em Planaltina - DF. Revista Brasileira de Botânica, São Paulo, v.8, p.131-142, 1985.

RIBEIRO, J.F.; PROENÇA, C.E.B.; ALMEIDA, S.P. Potencial frutífero de algumas espécies frutíferas nativas dos cerrados .In: CONGRESSO BRASILEIRO DE FRUTICULTURA, 8, 1986, Brasília, Anais v.2, p.491-500.

RIBEIRO, J.F.; SILVA, J.A., FONSECA, C.E.L. da. Espécies frutíferas da região do cerrado. In: DONADIO, L.C. (Coord.) Fruticultura tropical. Jaboticabal: FUNEP, 1992. 268p.

RIZZINI, C.T. Aspectos ecológicos da regeneração em algumas plantas do Cerrado. In: SIMPÓSIO SOBRE O CERRADO, 3., 1971, São Paulo, SP. Anais. São Paulo: Edgard Blucher, 1971. p. 61-64.

SANO, S.M. FONSECA; C.E.L. da F.; RIBEIRO, J.F.; et al. Folhação, floração, frutificação e crescimento inicial da cagaiteira. DF. Pesquisa Agropecuária Brasileira, Brasília, v.30, n.1, p.5$14,1995$.

SILVA, J.A.; SILVA, D.B.; JUNQUEIRA, N.T.V. et al. Coleta de sementes, produção de mudas e plantio de espécies frutíferas nativas dos cerrados: Informações exploratórias. Planaltina: EMBRAPA/CPAC, 1992.23p. (Documentos, 44).

TELLES, M. P. C., Diversidade genética e estrutura populacional de cagaiteira (Eugenia dysenterica DC) do Sudeste de Goiás. 2000. 63f. Tese (Dissertação) - Escola de Agronomia, Universidade Federal de Goiás, Goiânia, 2000. 